\title{
Expression of adrenomedullin in rats after spinal cord injury and intervention effect of recombinant human erythropoietin
}

\author{
LIANG ZHAO $^{1}$, YU JING ${ }^{2}$, LIN QU $^{1}$, XIANGWEI MENG ${ }^{1}$, YANG CAO $^{1}$ and HUIBING TAN ${ }^{3}$ \\ Departments of ${ }^{1}$ Orthopeadic Surgery and ${ }^{2}$ General Surgery, The First Affiliated Hospital of Jinzhou Medical University; \\ ${ }^{3}$ Department of Anatomy Teaching and Research, Jinzhou Medical University, Jinzhou, Liaoning 121000, P.R. China
}

Received May 13, 2016; Accepted September 19, 2016

DOI: $10.3892 / \mathrm{etm} .2016 .3832$

\begin{abstract}
The expression of adrenomedullin (ADM) in injured tissue of rat spinal cord was observed and the effect of recombinant human erythropoietin was analyzed. A total of 45 Sprague-Dawley rats were selected and divided into 3 equal groups including, a sham-operation group in which rats received an excision of vertebral plate; a spinal cord injury model group and a recombinant human erythropoietin group in which rats with spinal cord injury received a caudal vein injection of 300 units recombinant human erythropoietin after injury. Hematoxylin and eosin staining was performed to observe the spinal cord injury conditions. Immunohistochemical staining was performed to observe the expression of ADM. Pathologic changes in the group of recombinant human erythropoietin at various times were significantly less severe than those in the group of spinal cord injury model. The expression of ADM was increased particularly in the group of recombinant human erythropoietin $(\mathrm{P}<0.01)$. The improved Tarlov scores of the group of spinal cord injury model and the group of recombinant human erythropoietin were lower than those of the sham-operation group at 3, 6 and 9 days $(\mathrm{P}<0.01)$. Thus, the recombinant human erythropoietin is capable of alleviating the secondary injury of spinal cord. One of the mechanisms may be achieved by promoting the increase of ADM expression.
\end{abstract}

\section{Introduction}

Initially extracted from the pheochromocytoma tissue excised in a surgery (1), adrenomedullin (ADM) is a polypeptide

Correspondence to: Dr Yang Cao, Department of Orthopeadic Surgery, The First Affiliated Hospital of Jinzhou Medical University, No. 2 Renmin Road, Section 2, Jinzhou, Liaoning 121000, P.R. China E-mail: liangzhao160503@163.com

Dr Huibing Tan, Department of Anatomy Teaching and Research, Jinzhou Medical University, 40 Songpo Road 3, Jinzhou, Liaoning 121000, P.R. China

E-mail: liangzhao160503@163.com

Key words: spinal cord injury, adrenomedullin, recombinant erythropoietin, expression, rat sharing homology with calcitonin gene-related peptide and has functions of vascular dilation and diuretic activity (2). In a healthy individual, a considerable plasmatic concentration of ADM is involved in the regulation of plasma (3). In addition, as a bioactive peptide, ADM has extensive biological functions and is distributed in almost all tissues (4), particularly in normal adrenal medulla, heart, lung, kidney, blood plasma, and urine and is able to reduce high blood pressure, dilate blood vessels, pulmonary and renal arteries, and relieve bronchospasms (5).

ADM has multiple biological functions, including the regulation of internal secretion in organisms and inhibition of hyperplasia of vascular smooth muscles. Furthermore, ADM participates in multiple physiological accommodations in an autocrine and a paracrine manner as a circulating hormone. Currently, the pursuit of regulating measures for immune dysfunctions after trauma is becoming a field of interest. In the central nervous system, ADM and its mRNA are also widely distributed and located in neurons (6). Research has shown an increased expression of ADM after spinal cord injury, but there are few reports on its mechanism of expression, role and regulation (7).

For this reason, we aimed to detect the expression of $\mathrm{ADM}$ in a rat model of the spinal cord injury and attempted to discuss the mechanism of expression, effectiveness and regulation availability. The aim was to provide theoretical bases for clinical treatment of spinal cord injury by establishing a model of Sprague-Dawley (SD) rat for spinal cord injury and intervention of recombinant human erythropoietin.

\section{Materials and methods}

Animals. The experiment was completed in the Central Laboratory of the Department of Orthopaedics, The First Affiliated Hospital of Liaoning Medical University (Liaoning, China) from October, 2012 to March, 2013. A total of 45 Sprague-Dawley (SD) healthy rats aged 60 days and weighing 180-200 $\mathrm{g}$ were selected. The rats were provided by the Shanghai Laboratory Animal Center (SLAC) Co., Ltd. [animal license no. SCXK (Shanghai) 2012-0138; Shanghai, China]. The rats were divided into 3 groups randomly: Sham-operation group $(n=15)$, group of spinal cord injury model ( $\mathrm{n}=15)$, and group of recombinant human erythropoietin $(\mathrm{n}=15)$. 
Establishment of animal models. Pentobarbital sodium (5 g/1) was intraperitoneally injected as per $10 \mathrm{mg} / \mathrm{kg}$. Surgery was performed aseptically after anesthesia. An incision, $\sim 3 \mathrm{~cm}$ long, was made in the middle of the dorsal side to expose or injure the spinal cord section $\mathrm{T}_{10}$. Excision of vertebral plates was performed in the sham-operation group to expose the spinal cord. For the spinal cord injury model, a model of dorsal side injury of rat spinal cord was prepared using the Allen's WD method. The vertebral plate was excised to expose the spinal cord. A concave-type plastic sheet with the diameter of $2 \mathrm{~mm}$ was horizontally placed at the site of the spinal cord to be injured. A cylindrical metal bar was made and dropped freely and vertically in a thin glass delivery tube to establish an animal model of spinal cord injury. The metal bar weighed $10 \mathrm{~g}$ and was $10 \mathrm{~cm}$ high with injury energy of $10 \times 10(\mathrm{~g} \cdot \mathrm{cm})$. A model of dorsal side injury of rat spinal cord was prepared with the same method in the group of recombinant human erythropoietin. Caudal vein injection of $300 \mathrm{U} / \mathrm{kg}$ recombinant human erythropoietin was administered to the group of recombinant human erythropoietin at 1, 3, 5, 7 and 9 days after injury, respectively. An equivalent volume of normal saline was administered to the group of spinal cord injury after injury at the same time. The completeness of spinal dura mater was maintained and each layer was sutured in all the groups. The success marks of the injury animal model were tail wagging reflex and symmetric convulsion of both lower extremities (8).

The study was approved by the Animal Ethics Committee of Jinzhou Medical University.

Histopathological and immunohistochemical examination. All the groups of animals were anesthetized again at 3, 6 and 9 days after operation, respectively. A piece of spinal cord tissue with the approximate length of $1.0 \mathrm{~cm}$ was taken with the injured spinal cord section as the center, fixed in formaldehyde with the volume fraction of 0.3 , embedded with paraffin and serially sectioned to $5 \mu \mathrm{m}$ sections with a microtome, with 3 rats at each time. Regular hematoxylin and eosin staining and ADM immunohistochemical staining were performed. Regular hematoxylin and eosin staining was performed to observe edema, degeneration and necrosis, inflammatory cell infiltration in various groups of spinal cord tissues. The SABC method was used for immunohistochemical staining of ADM. The rabbit anti-rat ADM polyclonal antibody (1:100) and the SABC immunohistochemical staining reagent kit were purchased from Beijing Ruixiang Biotechnology Co., Ltd. (Beijing, China). Operations were in strict accordance with the instructions in the reagent kit. The expression of ADM was observed under a light microscope.

Image analysis. Five immunohistochemically-stained ADM sections were selected randomly from each rat. Five high-power fields were selected from the anterior angle to the relief angle of the grey matter of each section. The HMIAS-2000 high-definition color medical image-text analysis system was applied to determine the average gray value of the positive reactant of the ADM and to calculate the gray value.

Determination of motor function. The improved Tarlov scoring and the improved Rivlin and Tator tilting plate scoring were used to observe the recovery of the motor function of the lower limbs in rats at 3,6 and 9 days after operation, respectively (9).

Criteria for improved Tarlov scoring: 0 point: Complete paralysis, there is no response to acupuncture in lower limbs; 1 point: Complete paralysis, there is response to acupuncture in lower limbs but the limbs are not able to move; 2 points: The limbs are able to move but the rat cannot stand or stand steadily ( $<5 \mathrm{sec}$ ); 3 points: The rat can stand but cannot walk; 4 points: The rat can walk several steps but not steadily; 5 points: The rat can walk slowly but not flexibly and the walking has certain defects; 6 points: The rat can walk normally.

Improved Rivlin and Tator tilting plate method: The rat was placed on the same smooth board with its body axis perpendicular to the longitudinal axis of the tilting plate. The tilting plate was raised by $5^{\circ}$ each time and the maximum angle at which the rat could stay for $5 \mathrm{sec}$ was regarded as the functional value.

Statistical analysis. SPSS 13.0 statistical software (SPSS, Inc., Chicago, IL, USA) was used for statistical analysis. Data were expressed as mean \pm standard deviation. The $F$ test was used for variance analysis. $\mathrm{P}<0.05$ was considered to indicate a statistically significant difference.

\section{Results}

Quantitative analysis for experimental animals. A result analysis was conducted for all of the 45 rats with attrition value.

Result of hematoxylin and eosin staining. No significant abnormality was present in material sampled from the sham-operation group at various times. In the group of spinal cord injury model, 3 days after injury, a large number of erythrocytes were present within the grey matter; neural cells were swollen and exhibited a round shape; substantial karyopyknosis and karyorrhexis occurred; the border of grey matter disappeared; voids formed in the grey matter; 6 days after injury, erythrocytes fused into clusters; the border of grey matter was clear; the residual neurons decreased significantly and were slightly swollen; a small number of neutrophile granulocytes were present; 11 days after injury, the morphology of the residual neurons were basically normal. Pathological changes in the group of recombinant human erythropoietin at various times were significantly less severe than those in the group of spinal cord injury model (Fig. 1).

Result of immunohistochemical staining of ADM. The expression of ADM was primarily located in neurons. The expression of ADM was low at various times in the sham-operation group; compared with the sham-operation group, the number of neurons decreased and the expression of ADM was increased in the group of spinal cord injury model and group of recombinant human erythropoietin. The expression of ADM was increased particularly in the group of recombinant human erythropoietin. The expression was restored to normal at 6 and 9 days after injury.

Expression of ADM in various groups of rats at different time points. The difference in gray values of the sham-operation 
Table I. Comparison of gray values of positive reactants of ADM in various groups of rats at different times.

\begin{tabular}{lccc}
\hline & \multicolumn{3}{c}{ t(injury)/days } \\
\cline { 2 - 4 } Group & 3 & 6 & 9 \\
\hline Sham-operation & $0.328 \pm 0.007$ & $0.330 \pm 0.006$ & $0.325 \pm 0.007$ \\
Spinal cord injury model & $0.376 \pm 0.008^{\mathrm{a}}$ & $0.332 \pm 0.008$ & $0.331 \pm 0.009$ \\
Recombinant human erythropoietin & $0.412 \pm 0.010^{\mathrm{a}, \mathrm{b}}$ & $0.332 \pm 0.009$ & $0.334 \pm 0.007$ \\
\hline
\end{tabular}

Compared with the sham-operation group at the same time, ${ }^{\mathrm{a}} \mathrm{P}<0.01$; compared with the group of spinal cord injury model at the same time, ${ }^{\mathrm{b}} \mathrm{P}<0.01$. ADM, adrenomedullin.

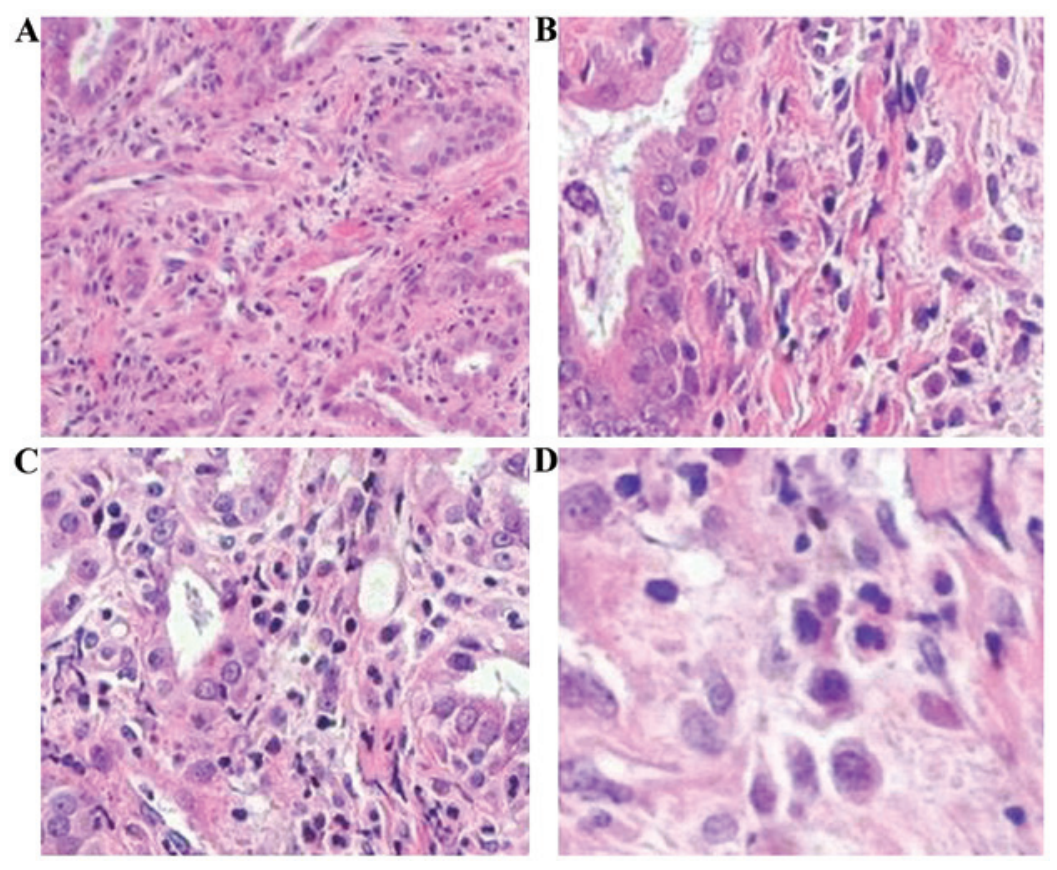

Figure 1. Result of immunohistochemical staining of ADM. (A) Sham-operation group at 3 days after injury; (B) group of spinal cord injury model at 3 days after injury; (C) group of recombinant human erythropoietin at 3 days after injury; (D) group of recombinant human erythropoietin at 4 days after injury.

group at various times was not significant indicating that an uninjured spinal cord did not affect the expression of ADM; the gray values of the group of spinal cord injury model and the group of recombinant human erythropoietin were higher than that of the sham-operation group at 3 days after injury and the gray value of the group of recombinant human erythropoietin was higher in the group of spinal cord injury model. Both differences were significant $(\mathrm{P}<0.01)$, which indicated that the expression of ADM after spinal cord injury was increased and the recombinant human erythropoietin was able to promote the high expression of ADM (Table I).

Result of improved Tarlov scoring. The improved Tarlov scores of the group of spinal cord injury model and the group of recombinant human erythropoietin were significantly lower than those of the sham-operation group at 3,6 and 9 days at the same time and the difference was significant $(\mathrm{P}<0.01)$. The improved Tarlov scores of the group of recombinant human erythropoietin were higher than those of the group of spinal cord injury model at 3,6 and 9 days at the same time and the difference was significant $(\mathrm{P}<0.05$; Table II).
Result of improved Rivlin and Tator scoring. The improved Rivlin and Tator scores of the group of spinal cord injury model and the group of recombinant human erythropoietin were significantly lower than those of the sham-operation group at 3, 6 and 9 days at the same time and the difference was significant $(\mathrm{P}<0.01)$; the improved scores of the group of recombinant human erythropoietin were higher than those of the group of spinal cord injury model at 3,6 and 9 days at the same time and the difference was significant $(\mathrm{P}<0.01$, Table III).

\section{Discussion}

ADM is an active peptide comprising 52 amino acids with many important biological functions. ADM is distributed in almost all tissues (10) and serves as a circulating hormone with autocrine and paracrine functions, in several tissues and organs such as blood plasma, adrenal gland, heart, kidney, central nervous system, and blood vessels. ADM participates in the regulation of blood vessel activities and stress response in organisms and exerts the compensatory regulation effect under many pathological conditions (11). Recently, the spinal 
Table II. Result of improved Tarlov scoring.

\begin{tabular}{llll}
\hline & & \multicolumn{3}{c}{$\mathrm{t}($ injury)/days } \\
\cline { 2 - 4 } Group & 3 & 6 & 9 \\
\hline Sham-operation & $7.36 \pm 0.67$ & $7.42 \pm 0.69$ & $7.43 \pm 0.66$ \\
Spinal cord injury model & $3.36 \pm 0.42$ & $2.97 \pm 0.38^{\mathrm{a}}$ & $3.64 \pm 0.47^{\mathrm{a}}$ \\
Recombinant human erythropoietin & $4.78 \pm 0.48^{\mathrm{a}, \mathrm{c}}$ & $4.83 \pm 0.51^{\mathrm{a} . \mathrm{b}}$ & $5.31 \pm 0.48^{\mathrm{a}, \mathrm{c}}$ \\
\hline
\end{tabular}

Compared with the sham-operation group at the same time, ${ }^{\mathrm{a}} \mathrm{P}<0.01$; compared with the group of spinal cord injury model at the same time, ${ }^{\mathrm{b}} \mathrm{P}<0.01,{ }^{\mathrm{c}} \mathrm{P}<0.05$.

Table III. Result of improved Rivlin and Tator scoring.

\begin{tabular}{llll}
\hline & & \multicolumn{3}{c}{ t(injury)/days } \\
\cline { 2 - 4 } Group & 3 & 6 & 9 \\
\hline Sham-operation & $67.16 \pm 8.13$ & $69.34 \pm 8.20$ & $70.28 \pm 8.17$ \\
Spinal cord injury model & $23.64 \pm 4.67^{\text {a }}$ & $26.16 \pm 4.81^{\text {a }}$ & $28.33 \pm 4.86$ \\
Recombinant human erythropoietin & $29.35 \pm 6.13^{\text {a,b }}$ & $35.26 \pm 6.32^{\text {a.b }}$ & $38.61 \pm 6.37^{\text {a,b }}$ \\
\hline
\end{tabular}

Compared with the sham-operation group at the same time, ${ }^{\mathrm{a}} \mathrm{P}<0.01$; compared with the group of spinal cord injury model at the same time, ${ }^{b} \mathrm{P}<0.01$.

cord injury has been raising general concerns due to its high occurrence rate, expenses and disability rate. In addition to primary mechanical injury, the spinal cord injury may also cause a series of secondary pathological changes. Currently, it is considered that the secondary spinal cord injury is a process of mutual cascading of many nerve biochemical and vascular mechanisms and continuous exacerbation. An irreversible injury within $4 \mathrm{~h}$ is a primary injury and various biochemical changes occurring after $4 \mathrm{~h}$ are secondary injuries and have certain reversibility. It is crucial to maximize recovery of spinal cord injury during the reversible period (12).

The expression of ADM increases in a compensatory manner after spinal cord injury and creates advantages for recovery of neurological functions. It plays an important role in recovery of an injured spinal cord (13). Its increasing and action mechanisms are: Firstly, local ischemic and hypoxia environment. The driving force for incremental regulation of ADM is hypoxia and the local ischemic and hypoxia environment after spinal cord injury provides conditions for incremental regulation of ADM that exists as a cytoprotection factor in the local ischemic and hypoxia environment and increases stability of the cytomembrane (14). Secondly, oxidative stress may increase the expression of ADM. Indeed, it has been shown that oxidative stress is one of the causes of organ damage under pathological conditions. Oxidative stress can increase the expression of ADM, and ADM can inhibit the oxidative stress. Therefore, ADM is determined as an endogenous antioxidant. The therapeutic effect of endogenous antioxidant (e.g., vitamin E) on spinal cord trauma has been demonstrated (15). Free radicals are cellular metabolites with high activity and removed by antioxidants (e.g., vitamin $\mathrm{C}$ and $\mathrm{E}$ ) or converted into oxygen and water by some enzymes (e.g., superoxide dismutase). After secondary spinal cord injury, depletion of endogenous antioxidant and decrease of superoxide dismutase are not able to remove the increasing free radicals, which can induce cell apoptosis and irreversible cell death (16). The increase of ADM is of great significance (17). The expression of ADM increases after spinal cord injury, which strengthens the oxidation resistance in spinal cord injury and alleviates the spinal cord injury via antioxidation (18). In the experiment, the expression of ADM is increased after spinal cord injury and further increased after intervention of recombinant human erythropoietin, which demonstrates that the ADM can be regulated. It is found through a pathological examination that neuronal injuries are significantly less severe than those in the group of spinal cord injury model at various times and the scores of neurological functions at 3,6 and 9 days after spinal cord injury are higher than those in the group of spinal cord injury model, which indicates the correlation between the increase of ADM and alleviation of the degree of spinal cord injury and promotion of the recovery of neurological functions.

Recombinant human erythropoietin is a member of the cytokine superfamily. In addition to the function of promoting proliferation, differentiation, and maturation of progenitor erythrocytes, it also has anti-vasospasm, anti-apoptosis and anti-inflammatory functions (19). The recombinant human erythropoietin and its receptor exist in the hemopoietin system, the central nervous system, and the peripheral nervous system and can protect, not only cerebral injuries due to various causes, but also the acute spinal cord injury and secondary injury (19). The recombinant human erythropoietin is capable of protecting injuries of neurocytes and neural cells due to various causes 
such as excitotoxicity and lack of neurotrophic factors (20). Hypoxia can promote the expression of recombinant human erythropoietin and it has been demonstrated that the recombinant human erythropoietin can promote survival of neurocytes under hypoxia (21). In the experiment, the recombinant human erythropoietin was used to treat the spinal cord injury of rats. It is observed through a pathological examination that neuronal injuries are significantly less severe than those in the group of recombinant human erythropoietin at various times and the scores of neurological functions are higher than those in the group of spinal cord injury model, which indicates the therapeutical effect of recombinant human erythropoietin.

In conclusion, the ADM increases compensatory effects after spinal cord injury and the ADM promotes the recovery of neurological functions of the spinal cord primarily by antioxidation. The recombinant human erythropoietin can promote further high expression of the ADM. The correlation between the therapeutical effect of the recombinant human erythropoietin and the high expression of ADM suggested a mechanisms of treating spinal cord injury. Thus, treatment with recombinant human erythropoietin may be expected as an effective method for treatment of spinal cord injury.

\section{References}

1. Kitamura K, Kangawa K, Kawamoto M, Ichiki Y, Nakamura S, Matsuo $\mathrm{H}$ and Eto T: Adrenomedullin: A novel hypotensive peptide isolated from human pheochromocytoma. Biochem Biophys Res Commun 192: 553-560, 1993.

2. Tazaki M, Endoh T, Kobayashi H, Nobushima H, Shibukawa Y, Tsumura M, Sato M, Ubaidus S and Sueishi K: Adrenomedullin facilitates calcium channel currents in osteoblasts. Bull Tokyo Dent Coll 53: 203-206, 2012.

3. Yuan M, Wang Q, Li C, Tao L, Zhang H, Wang H, Zhang Y and Ren J: Adrenomedullin in vascular endothelial injury and combination therapy: Time for a new paradigm. Curr Vasc Pharmacol 13: 459-466, 2015.

4. Yoshizawa T, Sakurai T, Kamiyoshi A, Ichikawa-Shindo Y, Kawate H, Iesato Y, Koyama T, Uetake R, Yang L, Yamauchi A, et al: Novel regulation of cardiac metabolism and homeostasis by the adrenomedullin-receptor activity-modifying protein 2 system. Hypertension 61: 341-351, 2013.

5. Sakimoto S, Kidoya H, Kamei M, Naito H, Yamakawa D, Sakaguchi H, Wakabayashi T, Nishida K and Takakura N: An angiogenic role for adrenomedullin in choroidal neovascularization. PLoS One 8: e58096, 2013.

6. Dai X, Ma W, He XJ and Jha RK: Elevated expression of adrenomedullin is correlated with prognosis and disease severity in osteosarcoma. Med Oncol 30: 347, 2013.

7. Hong Y, Liu Y, Chabot J-G, Fournier A and Quirion R: Upregulation of adrenomedullin in the spinal cord and dorsal root ganglia in the early phase of CFA-induced inflammation in rats. Pain 146: 105-113, 2009
8. Saxena T, Deng B, Stelzner D, Hasenwinkel J and Chaiken J: Raman spectroscopic investigation of spinal cord injury in a rat model. J Biomed Opt 16: 027003, 2011.

9. Jiang Y, Lv H, Huang S, Tan H, Zhang Y and Li H: Bone marrow mesenchymal stem cells can improve the motor function of a Huntington's disease rat model. Neurol Res 33: 331-337, 2011.

10. Brouwers FP, de Boer RA, van der Harst P, Struck J, de Jong PE, de Zeeuw D, Gans RO, Gansevoort RT, Hillege HL, van Gilst WH, et al: Influence of age on the prognostic value of mid-regional pro-adrenomedullin in the general population. Heart 98: 1348-1353, 2012.

11. Tsuchiya K, Hida K, Hida Y, Muraki C, Ohga N, Akino T, Kondo T, Miseki T, Nakagawa K, Shindoh M, et al: Adrenomedullin antagonist suppresses tumor formation in renal cell carcinoma through inhibitory effects on tumor endothelial cells and endothelial progenitor mobilization. Int J Oncol 36: 1379-1386, 2010.

12. Shibasaki I, Nishikimi T, Mochizuki Y, Yamada Y, Yoshitatsu M, Inoue Y, Kuwata T, Ogawa H, Tsuchiya G, Ishimitsu T, et al: Greater expression of inflammatory cytokines, adrenomedullin, and natriuretic peptide receptor- $\mathrm{C}$ in epicardial adipose tissue in coronary artery disease. Regul Pept 165: 210-217, 2010.

13. Marinoni E, Pacioni K, Sambuchini A, Moscarini M, Letizia C and DI Iorio R: Regulation by hypoxia of adrenomedullin output and expression in human trophoblast cells. Eur J Obstet Gynecol Reprod Biol 154: 146-150, 2011.

14. Kim SM, Kim JY, Lee S and Park JH: Adrenomedullin protects against hypoxia/reoxygenation-induced cell death by suppression of reactive oxygen species via thiol redox systems. FEBS Lett 584: 213-218, 2010.

15. Oz Oyar E, Korkmaz A, Kardesş O and Omeroğlu S: Aortic cross-clamping-induced spinal cord oxidative stress in rabbits: The role of a novel antioxidant adrenomedullin. J Surg Res 147: 143-147, 2008.

16. Pfeil U, Aslam M, Paddenberg R, Quanz K, Chang CL, Park JI, Gries B, Rafiq A, Faulhammer P, Goldenberg A, et al: Intermedin/adrenomedullin-2 is a hypoxia-induced endothelial peptide that stabilizes pulmonary microvascular permeability. Am J Physiol Lung Cell Mol Physiol 297: L837-L845, 2009.

17. Arduini A, Escobar J, Vento M, Escrig R, Quintás G, Sastre J, Saugstad OD and Solberg R: Metabolic adaptation and neuroprotection differ in the retina and choroid in a piglet model of acute postnatal hypoxia. Pediatr Res 76: 127-134, 2014.

18. Yen DH, Chen LC, Shen YC, Chiu YC, Ho IC, Lou YJ, Chen IC and Yen JC: Protein kinase A-dependent neuronal nitric oxide synthase activation mediates the enhancement of baroreflex response by adrenomedullin in the nucleus tractus solitarii of rats. J Biomed Sci 18: 32, 2011.

19. Ning B, Zhang A, Song H, Gong W, Ding Y, Guo S, Zhao Y, Jiang $\mathrm{J}$ and Jia T: Recombinant human erythropoietin prevents motor neuron apoptosis in a rat model of cervical sub-acute spinal cord compression. Neurosci Lett 490: 57-62, 2011.

20. Huang H, Fan S, Ji X, Zhang Y, Bao F and Zhang G: Recombinant human erythropoietin protects against experimental spinal cord trauma injury by regulating expression of the proteins MKP-1 and p-ERK. J Int Med Res 37: 511-519, 2009.

21. Wang H,Liu ZL, Zhuang XT, Wang MF and Xu L: Neuroprotective effect of recombinant human erythropoietin on optic nerve injury in rats. Chin Med J (Engl) 122: 2008-2012, 2009. 